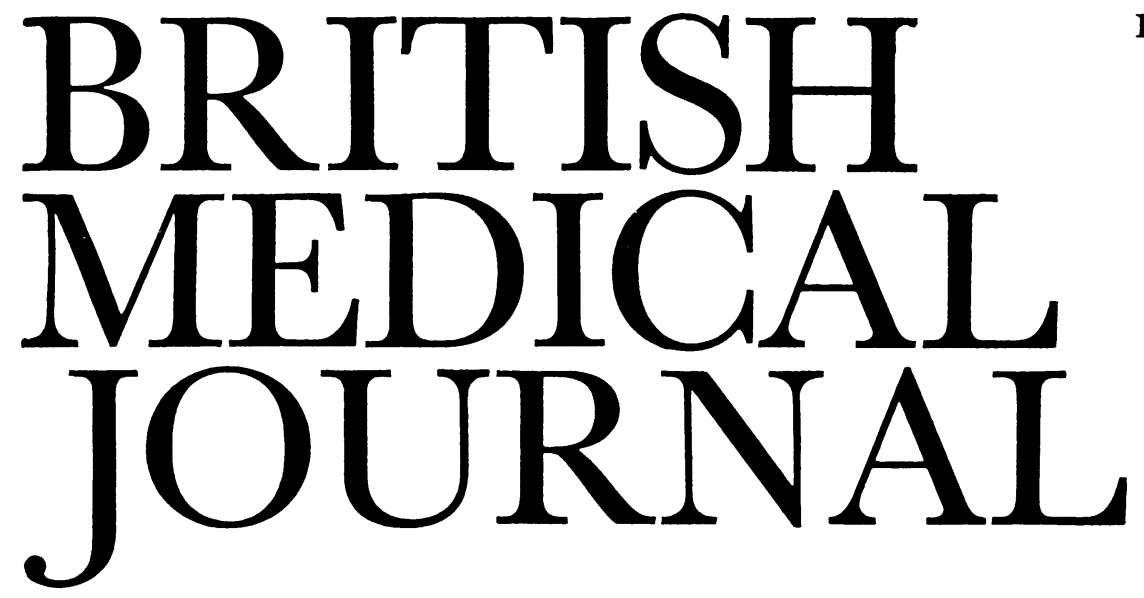

LONDON, SATURDAY 28 FEBRUARY 1981

\title{
Febrile convulsions: long-term treatment
}

Febrile convulsions are common but the use of prophylactic anticonvulsants has been controversial. Opinions vary between giving anticonvulsants to all or selected children who have had febrile convulsions and giving them to none. Those who advocate long-term drugs point to the possible sequelae of another attack. Practical guidelines are now emerging from large long-term studies ${ }^{1}$ and consensus opinions. ${ }^{2}$

Febrile convulsions are classed as simple when they occur in infants who are developing normally and are aged between 1 year and 5 years, have no focal features, and the fit lasts less than 15 minutes. ${ }^{1}$ There is no evidence of a central nervous system infection or a metabolic cause. In the collaborative study of Nelson and Ellenberg in 1706 children $^{1}$ no patient with a simple febrile convulsion was found to have a serious subsequent neurological disorder or intellectual impairment. Only $2 \cdot 2 \%$ of children with simple febrile convulsions will eventually develop convulsions without fever, ${ }^{1}$ and treatment of this group with adequate prophylaxis will not decrease this risk. ${ }^{2}$ Children with simple febrile convulsions make up twothirds of children with convulsions and fever and as their prognosis is excellent no drugs are indicated. ${ }^{3}$ Nevertheless, repeated simple convulsions, though not dangerous, are frightening for both the parents and the child. Only one-third of children who have simple febrile convulsions will have another attack, but $10 \%$ will have multiple episodes, and anticonvulsants should be considered for that group. ${ }^{1}$ Indications for treatment have been confused in the past because of the mixing together of patients with poor prognosis with those of good prognosis.

Who else should receive drugs and what drugs be given? There has never been controversy about giving anticonvulsant drugs to children who have previous neurological disorders or developmental delay. Status epilepticus occurs most frequently with the first convulsion (and therefore prophylactic treatment does not help). Children with febrile convulsions who later develop temporal lobe epilepsy usually have poor prognostic features that may be recognised in most cases. ${ }^{3}$ Long-term neurological problems are likely to be an expression of unrecognised neurological abnormalities or to be the result of a prolonged or complex convulsion occurring in a normal child with fever. ${ }^{3}$ The children who need drugs are those with complex convulsions which are characterised by focal features, a duration of over 15 minutes, onset before the age of 1 year, or a family history of febrile seizures. Children with any of the above adverse features have a $10 \%$ risk of later developing convulsions without fever, including temporal lobe epilepsy. ${ }^{1}$

Effective prophylactic treatment of febrile convulsions resulting in a decrease in the recurrence rate has been achieved using phenobarbitone or sodium valproate and monitoring drug concentrations in the blood. ${ }^{4}$ Phenobarbitone $4-6 \mathrm{mg} / \mathrm{kg} /$ day has been well tried and no serious long-term side effects have been reported. Nevertheless, $40-50 \%$ of children taking phenobarbitone develop problems which include irritability, hyperactivity, negativistic behaviour, and rashes. These are severe enough to warrant withdrawal of treatment in $20 \%$ of cases and result in a high incidence of poor compliance. ${ }^{5}$

Therapeutic serum concentrations of phenobarbitone are obtained only 48 hours after oral administration, and even after intramuscular administration a therapeutic effect is delayed for up to 24-36 hours. Since around half of all febrile convulsions occur in the first 24 hours of a febrile illness, prophylactic phenobarbitone must be given continuously. ${ }^{3}$

Sodium valproate was first introduced in the long-term management of febrile convulsions in $1975 .{ }^{6}$ Since then it has been shown to be at least as effective as phenobarbitone ${ }^{4}$ ? in preventing repeated convulsions and the incidence of side effects is low-gastrointestinal disturbances, gain in weight, and alopecia. Much more rarely, however, serious side effects have been recorded, including liver failure and pancreatitis. ${ }^{18}$ These complications prevent sodium valproate from being widely used as the first choice for prophylactic management.

At present, then, phenobarbitone should be regarded as the drug of choice in preventing recurrent febrile convulsions. Should phenobarbitone not be tolerated in therapeutic doses then a change to sodium valproate $20-30 \mathrm{mg} / \mathrm{kg} /$ day in two divided doses is reasonable. Convulsions precipitated by fever in the presence of previous developmental and neurological abnormalities or in the presence of a family history of afebrile convulsions or with focal features should also be treated primarily with sodium valproate, which is more effective than phenobarbitone in preventing recurrences in this group. ${ }^{9}$

With this regimen of selective medication, almost two-thirds of children with febrile convulsions may be excluded from drug treatment. Parents need to be taught how to control future fevers and manage possible further convulsions. The initial convulsion warrants admission to hospital, especially if occurring under the age of 3, when signs and symptoms of meningitis may be far from obvious. Subsequent simple 
convulsions can safely be managed at home. Parents need, however, an adequate explanation of the condition with an optimistic outlook in order to allay their reasonable anxiety.

1 Nelson KB, Ellenberg JH. Predictors of epilepsy in children who have experienced febrile seizures. N Engl F Med 1976;295:1029-33.

2 National Institutes of Health Consensus Statement. Febrile seizures : longterm management of children with fever-associated seizures. $\mathrm{Br} \mathrm{Med} \mathcal{F}$ $1980 ; 281: 277-9$.

${ }^{3}$ Fishman MA. Febrile seizures: the treatment controversy. 7 Pediatr 1979; 94:177-84.

${ }^{4}$ Wallace SJ, Smith JA. Successful prophylaxis against febrile convulsions with valproic acid or phenobarbitone. $B r \operatorname{Med} \mathcal{F} 1980 ; 280: 353-4$.

5 Wolf SM, Forsythe A. Behavior disturbance, phenobarbital, and febrile seizures. Pediatrics 1978;61:728-31.

6 Cavazzuti GB. Prevention of febrile convulsions with dipropylacetate (Depakine (R)). Epilepsia 1975;16:647-8.

7 Ngwane E, Bower B. Continuous sodium valproate or phenobarbitone in the prevention of "simple" febrile convulsions. Comparison by a doubleblind trial. Arch Dis Child 1980;55:171-4.

${ }^{8}$ Rall TW, Schleifer LS. Drugs effective in the therapy of the epilepsies. In: Goodman LS, Gilman A, eds. The pharmacological basis of therapeutics. London: Baillière Tindall, 1980:448-74.

9 Jeavons PM, Clark JE. Sodium valproate in treatment of epilepsy. Br Med f 1974 ;ii :584-6.

\section{They are still with us}

Half-remembered clinical entities (except where they intrude into Western outpatient clinics or puzzle general practitioners), the major tropical diseases are still with us; in fact, they are probably more prevalent today than ever. The annual reports of the Special Programme for Research and Training in Tropical Diseases ${ }^{1}$ may not be the most dramatic of documents, but they should be required reading for all concerned with larger matters of public health.

Malaria, schistosomiasis, filariasis (including onchocerciasis), trypanosomiasis (both African sleeping sickness and Chagas's disease), leishmaniasis (both visceral and mucocutaneous), and leprosy are the diseases selected some five years ago by the World Health Organisation for special attack. Smallpox was then well on the way to eradication, and yaws seemed to have been eliminated. Poliomyelitis, cholera, and yellow fever could be contained, and tuberculosis could be treated. But for long these six major diseases had resisted piecemeal efforts at control. The early euphoria that had attended valiant efforts at controlling malaria had evaporated into pessimism. A new initiative was needed; it was provided in the imaginative protocol adopted by the World Health Organisation, endorsed by its member governments, and generously supported by supranational funding agencies, governments, and individual and corporate donors.

What is the picture today? The resurgence of malaria in south-east Asia and elsewhere is posing virtually intractable problems. Insecticide-resistant anopheline vectors and drugresistant plasmodial parasites demand a fundamental rethinking of the whole strategy of control. Until an adequate health infrastructure becomes more generally available attempts at vector control and mass medication will continue to be ineffective, especially in the presence of financial constraints and some lack of official determination.

Schistosomiasis, too, is spreading, as optimum conditions for the multiplication of the snails, which are the intermediate host, are provided by irrigation canals and hydroelectric engineering works. Forewarned is not, regrettably, always forearmed. Persistence and extension of the disease are intimately linked with social habits (conservatively resistant to change) and the difficulty of interrupting the cycle of transmission at any stage.

While considerable progress has been made by the ambitious onchocerciasis control programme in West Africa, economic returns on the huge capital investment have yet to appear. Bancroftian filariasis remains virtually unchecked. The disruption of rural medical services in much of subsahelan Africa has had devastating consequences on control programmes in trypanosomiasis, with reactivation and extension of old foci in Zaïre and other countries. The prevalence of Chagas's disease in South America will probably not be reduced until the standards of rural housing and domestic hygiene improve. Leishmaniasis, both visceral and mucocutaneous, is perhaps the least important of the six diseases singled out in the World Health Organisation programme; but, in terms of the danger of explosive epidemics (as in India) and diagnostic difficulties in skin clinics in the West, its place on the list may readily be justified.

Leprosy is now presenting considerable control problems in many countries. The threat of sulphone resistance is becoming more widespread, and "persister" organisms, dormant and drug-sensitive, may cause clinical and bacteriological relapse in patients who had suffered from multibacillary leprosy rendered quiescent by apparently adequate treatment, admittedly monotherapeutic.

Far from being "on the way out," therefore, these six formidable foes are still with us, and will continue to menace vast sections of dwellers in (and visitors to) the countries of the medicogeographical tropics. The setting is scarcely propitious: undernutrition, overpopulation, poverty, low standards of hygiene, lack of adequate medical infrastructure, political instability, and many other social and economic factors militate against the desirable control measures. All strength, then, to the WHO Research and Training Programme as it pursues its huge task, not only of encouraging and coordinating the use of modern investigative tools and techniques for research into these tropical scourges and their treatment and prevention, but also of bridging the gap between what is known and what is being done. High-quality laboratory-based contributions to the biomedical sciences must be matched by good field work and epidemiological research if control measures are to achieve any success. Meanwhile, there can be no more challenging and rewarding task today than that of research and training in tropical diseases.

${ }^{1}$ United Nations Development Programme/World Bank/World Health Organisation. Special programme for research and training in tropica diseases. Fourth Annual Report. Geneva: UNDP/World Bank/WHO, 1980.

\section{Interferon production by genetic engineering}

The mystique which surrounds interferon is in large part caused by its scarcity. In the early 1960s the amounts needed to change the course of viral infections in animals or man were found to be several thousands of times greater than could be easily prepared in the laboratory. Cantell has shown that a large-scale process is feasible by diverting buffy coats from large volumes of donated blood ${ }^{1}$; his group has provided most of the interferon for the clinical trials reported from around the world. 